\title{
Oxymatrine inhibits microglia activation via HSP60-TLR4 signaling
}

\author{
FEIJIA DING $^{1 *}$, YUNHONG LI $^{1 *}$, XIAOLIN HOU ${ }^{2 *}$, RUI ZHANG ${ }^{1}$, SHUTING HU $^{1}$ and YIN WANG ${ }^{1}$ \\ ${ }^{1}$ Department of Neurobiology, Ningxia Key Laboratory of Cerebrocranial Diseases, Ningxia Medical University; \\ ${ }^{2}$ Department of Neurology, The General Hospital of Ningxia Medical University, Yinchuan, Ningxia 750004, P.R. China
}

Received June 10, 2016; Accepted September 16, 2016

DOI: 10.3892/br.2016.776

\begin{abstract}
Oxymatrine (OMT) is an alkaloid extracted from Sophora flavescens, which has broad anti-inflammatory, antitumor and immunosuppressant actions. However, the underlying molecular mechanisms have remained elusive. Heat shock protein 60 (HSP60) has recently been shown to have an important role in autoimmune reactions. The present study aimed to investigate whether OMT exerts its anti-inflammatory effects by inhibiting microglial activation and examined the role of HSP60 in this process. Western blot analysis and ELISA showed that OMT decreased the expression and release of HSP60 by LPS-activated BV2 cells. The expression of heat shock factor 1, the transcription factor of HSP60, was also suppressed by OMT. Extracellular HSP60 has been previously indicated to induce microglial apoptosis through the Toll-like receptor (TLR)-4 pathway. Flow cytometric analysis demonstrated that LPS treatment induced apoptosis of BV2 cells, which was inhibited by OMT in parallel with inhibition of LPS-induced expression of TLR-4. Furthermore, OMT was shown to suppress the levels of myeloid differentiation factor (MYD)88, nuclear factor (NF)- $\mathrm{B}$, caspase-3, inducible nitric oxide synthase, tumor necrosis factor- $\alpha$, interleukin (IL)-1 $\beta$ and IL-6. In light of these results, it was concluded that OMT may exert its neuroprotective effects via HSP60/TLR-4/MYD88/NF- $\mathrm{B}$ signaling pathways to inhibit microglial activation. OMT may therefore offer substantial therapeutic potential for treating neurodegenerative diseases associated with microglial activation.
\end{abstract}

Correspondence to: Professor Yin Wang, Department of Neurobiology, Ningxia Key Laboratory of Cerebrocranial Diseases, Ningxia Medical University, 1160 Shengli Street, Yinchuan, Ningxia 750004, P.R. China

E-mail: yin-wang@hotmail.com

*Contributed equally

Key words: oxymatrine, heat shock protein 60, Toll-like receptor 4, BV2 microglia

\section{Introduction}

Microglial cells, the residential macrophages of the brain, have a pivotal role in the central nervous system's (CNS) innate immune response and act as the first line of defense against microorganism invasion and injury (1). Lipopolysaccharide (LPS) activates microglia to secrete various pro-inflammatory and cytotoxic factors, including tumor necrosis factor (TNF)- $\alpha$, interleukin (IL)-1 $\beta$ and heat shock protein 60 (HSP60) $(2,3)$. These factors are responsible for several neurodegenerative diseases, including Parkinson's and Alzheimer's disease as well as amyotrophic lateral sclerosis (4-6). Thus, controlling microglia activation may represent an effective therapeutic strategy against neurodegenerative diseases.

Oxymatrine (OMT) is a traditional Chinese medicine derived from the root of Sophora flavescens. Previous studies have revealed that OMT has numerous pharmacological effects, including anti-inflammatory, antitumor and antioxidant activities $(7,8)$. Several studies have also indicated a neuroprotective role of OMT. OMT has been reported to protect neurons by downregulating 12/15-lipooxygenase, phospho-p38 mitogen-activated protein kinase and cytosolic phospholipase A2 (9). However, the neuroprotective effects of OMT have remained to be fully elucidated.

HSP60 has pro-survival as well as pro-apoptotic functions. Under stress conditions, microglial cells highly express HSP60 and release it into the extracellular space, causing cell death through binding Toll-like receptor (TLR)-4 (10). The present study aimed to assess the neuroprotective effects of OMT in LPS-induced BV2 microglial cells as well as to identify the possible mechanistic involvement of HSP60. It was revealed that OMT likely suppressed microglial activation via the HSP60/TLR-4/myeloid differentiation factor (MYD)88/nuclear factor (NF)-кB signaling pathway.

\section{Materials and methods}

Chemicals. The BV2 murine microglial cell line was purchased from the Cell Bank of the Chinese Academy of Sciences (Shanghai, China). LPS was purchased from Sigma-Aldrich (St. Louis, MO, USA). OMT was from Guanyu Bio-tech (Xi'an, China). Antibodies against caspase-3 (cat. no. 9664; 1:2,000 dilution), MYD88 (cat. no. 4283; 1:1,000 dilution) and TLR-4 (cat. no. 2219; 1:1,000 dilution) were from Cell 
Signaling Technology, Inc. (Beverly, MA, USA). Anti-NF-кB (cat. no. ab131485; 1:200 dilution) and anti-inducible nitric oxide synthase (iNOS) antibodies (cat. no. ab3523; 1:200 dilution) were from Abcam (Cambridge, MA, USA). Antibodies against HSP60 (ADI-SPA-806-D, 1:1,000) and heat shock factor (HSF)-1 (cat. no. ADI-SPA-950-D; 1:1,000 dilution) were purchased from Stressgen (San Diego, CA, USA). Anti- $\beta$-actin antibody (cat. no. ZM-0001; 1:1,000 dilution) was purchased from Zhongshan Goldenbridge Bio. IL-6, IL-1 $\beta$ and TNF- $\alpha$ ELISA kits were acquired from Xinbosheng Biotechnology Inc. (Shenzhen, China). Enhanced chemiluminescence (ECL) and bicinchoninic acid (BCA) kits were from Pierce (Thermo Fisher Scientific, Inc., Waltham, MA, USA). Dulbecco's modified Eagle's medium (DMEM) and fetal bovine serum (FBS) were from Gibco (Thermo Fisher Scientific, Inc). The Cell Counting Kit-8 (CCK-8) and the Annexin V-fluorescein isothiocyanate (FITC)/propidium iodide (PI) apoptosis detection kit were obtained from Beibo (Shanghai, China).

Cell culture. BV2 cells were maintained in DMEM with $10 \% \mathrm{FBS}$ and grown at $37^{\circ} \mathrm{C}$ in a humidified atmosphere containing 5\% $\mathrm{CO}_{2}$. OMT was dissolved in DMEM. Cells were pre-treated with LPS $(1 \mu \mathrm{g} / \mathrm{ml})$ for $30 \mathrm{~min}$, followed by addition of different concentrations of OMT. After $24 \mathrm{~h}$, the supernatant of the cells was obtained for ELISA, and cells were collected, a proportion of which were lysed using a total protein extraction kit (KeyGen Biotech, Jiangsu, China) for subsequent experiments.

Cell viability assay. Cell viability was evaluated using the CCK-8 assay. Cells were seeded into 96-well microtiter plates at $5 \times 10^{4}$ cells/well and pre-treated with LPS $(1 \mu \mathrm{g} / \mathrm{ml})$ for $30 \mathrm{~min}$, followed by treatment with various concentrations of $\operatorname{OMT}(0,1,10,20,50$ or $100 \mu \mathrm{g} / \mathrm{ml})$. After $24 \mathrm{~h}$ of incubation, CCK-8 solution was added to each well, followed by incubation for $2 \mathrm{~h}$. The color intensity of the metabolized CCK- 8 stain was determined by measuring the absorbance at $450 \mathrm{~nm}$ with an immunoreader (PR4100; Bio-Rad Laboratories, Inc., Hercules, CA, USA). Cell viability was expressed as a percentage of the control group (untreated). Every experiment was repeated three times.

ELISA. BV2 cells were pre-treated with LPS $(1 \mu \mathrm{g} / \mathrm{ml})$ for $30 \mathrm{~min}$, followed by treatment with 0 or $20 \mu \mathrm{g} / \mathrm{ml}$ OMT for $24 \mathrm{~h}$ and subsequent collection of the culture medium. In a control group, the cells were left untreated. The levels of TNF- $\alpha$, IL-6, IL-1 $\beta$ and HSP60 in the culture medium were determined by ELISA kits according to the manufacturer's instructions. The absorbance was measured at $450 \mathrm{~nm}$ on a microplate reader (Bio-Rad Laboratories, Inc.).

Western blot analysis. To determine the expression of certain proteins, BV2 cells were pre-treated with LPS $(1 \mu \mathrm{g} / \mathrm{ml})$ for $30 \mathrm{~min}$, followed by treatment with 0 or $20 \mu \mathrm{g} / \mathrm{ml}$ OMT for $24 \mathrm{~h}$, and protein was extracted using a lysis buffer containing $0.1 \%$ Triton X-100, $2 \%$ sodium dodecyl sulfate (SDS), $5 \%$ glycerol, $5 \mathrm{mM}$ ethylenediaminetetraacetate, $150 \mathrm{mM} \mathrm{NaCl}$ and $20 \mathrm{mM}$ Tris (pH 7.5) (Bioscience, Shanghai, China). Following incubation at $4^{\circ} \mathrm{C}$ for $30 \mathrm{~min}$, the lysates were centrifuged at $8,050 \mathrm{x} \mathrm{g}$ at $4^{\circ} \mathrm{C}$ for $30 \mathrm{~min}$. The BCA kit was then used to

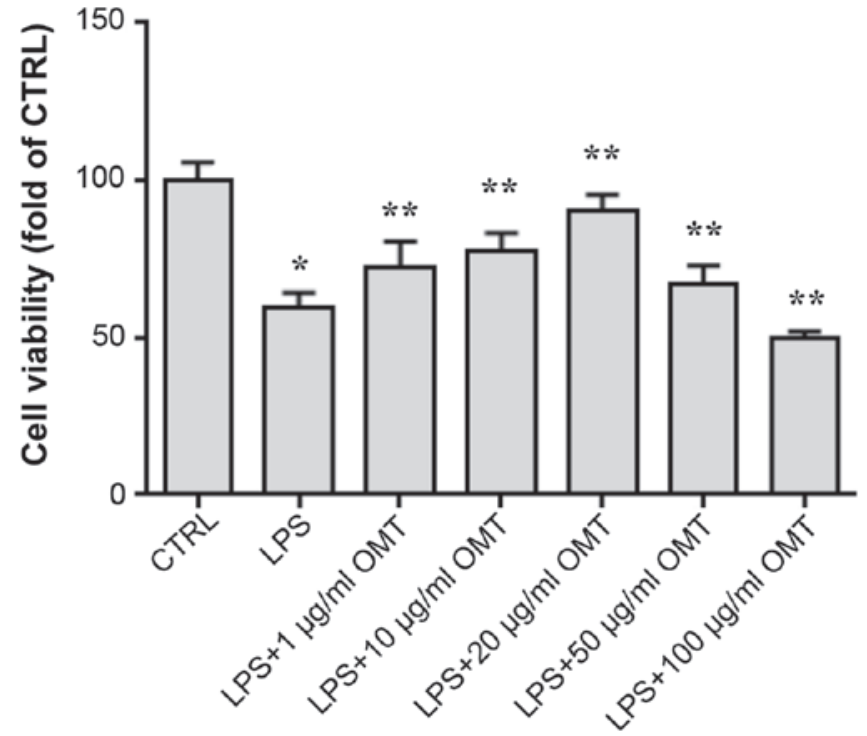

Figure 1. Effects of OMT on cell viability. BV2 cells were pre-treated with LPS $(1 \mu \mathrm{g} / \mathrm{ml})$ for $30 \mathrm{~min}$, followed by addition of various concentrations of $\operatorname{OMT}(1,10,20,50$ or $100 \mu \mathrm{g} / \mathrm{ml})$ and incubation for $24 \mathrm{~h}$. Values are expressed as the mean \pm standard error of the mean of three independent experiments. ${ }^{*} \mathrm{P}<0.05$ (LPS group vs. CTRL) or ${ }^{* *} \mathrm{P}<0.05$ (OMT groups vs. LPS group). CTRL, control; LPS, lipopolysaccharide; OMT, oxymatrine.

determine the protein concentration. Protein denaturation was then performed in a sample buffer containing 2-mercaptoethanol and bromophenol blue (KeyGen Biotech) for $10 \mathrm{~min}$ at $95^{\circ} \mathrm{C}$. Equal amounts of protein were resolved by $10 \%$ SDS-polyacrylamide gel electrophoresis and transferred onto a polyvinylidene difluoride membrane (EM Millipore, Billerica, MA, USA). The membranes were blocked with 5\% milk in Tris-buffered saline containing Tween-20 and incubated at $4{ }^{\circ} \mathrm{C}$ overnight with the indicated antibodies, including anti-iNOS, -TLR-4, -heat shock factor 1 (HSF-1), -NF-кВ, -HSP60, -caspase-3, -MYD88 and - $\beta$-actin. After washing with phosphate-buffered saline (PBS), the membranes were incubated with anti-mouse (cat. no. ZB-2305; 1:5,000) or anti-rabbit (cat. no. ZB-2301; 1:5,000) secondary antibodies (Zhongshan Goldenbridge Bio) at room temperature for $75 \mathrm{~min}$. The proteins were then visualized using the ECL kit and membranes were exposed to X-ray film. The blotting results were semi-quantified using Quantity One software, version 4.6.9 (Bio Rad Laboratories, Inc.).

Flow cytometry. BV2 cells were divided into three groups: Control group, LPS group [treated with LPS $(1 \mu \mathrm{g} / \mathrm{ml})$ for $24 \mathrm{~h}$ ] and the LPS+OMT group [treated with LPS $(1 \mu \mathrm{g} / \mathrm{ml})$ for $30 \mathrm{~min}$ and subsequently with OMT $(20 \mu \mathrm{g} / \mathrm{ml})]$. The cells were then collected and washed twice with cold PBS. Following centrifugation at $126 \mathrm{xg}$ for $4 \mathrm{~min}$ at room temperature, cells were re-suspended in $400 \mu \mathrm{l}$ binding buffer. After incubation with $5 \mu \mathrm{l}$ Annexin V-FITC for $15 \mathrm{~min}, 10 \mu \mathrm{l}$ PI was added, followed by incubation for $5 \mathrm{~min}$ in the dark. The apoptotic rate was measured by a FACSCalibur flow cytometer (BD Biosciences, Franklin Lakes, NJ, USA).

Statistical analysis. Values are expressed as the mean \pm standard error of the mean. One-way analysis of variance, followed 

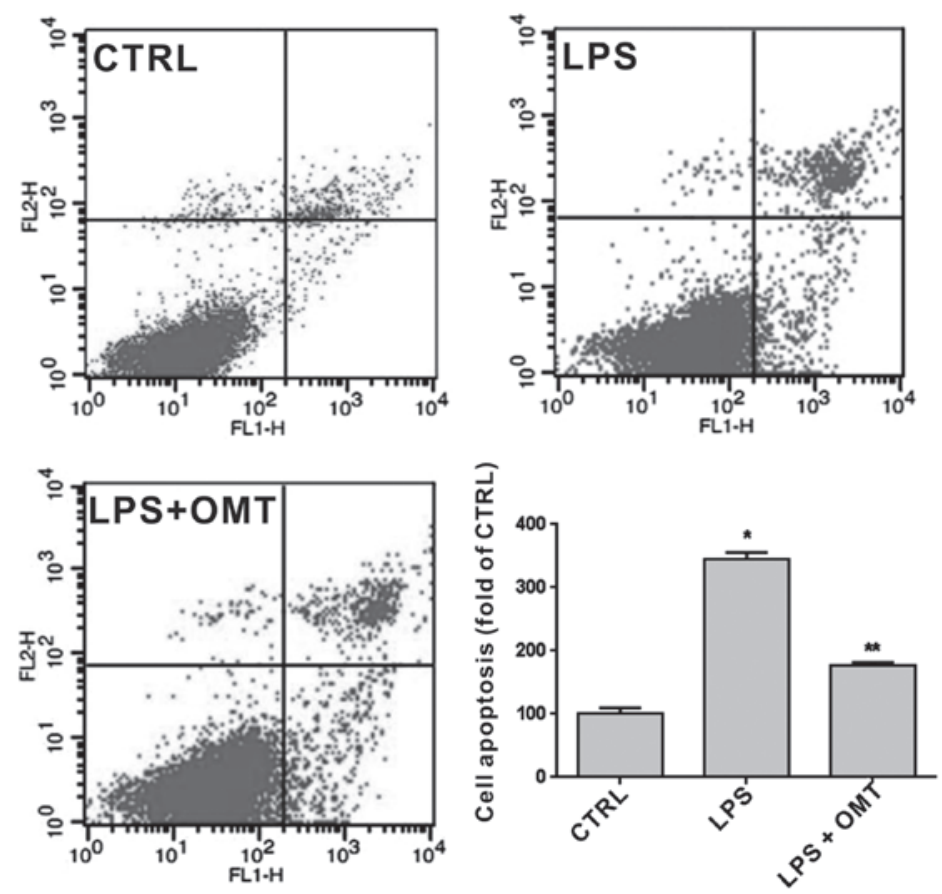

Figure 2. BV2 cell apoptosis assessed by flow cytometry. Live cells are displayed in the lower left quadrant (FITC-/PI-), early apoptotic cells in the lower right quadrant (FITC+/PI-) and late apoptotic cells in the upper right quadrant (FITC+/PI+). The results showed that OMT reduced LPS-induced apoptosis of BV2 cells. Values are expressed as the mean \pm standard error of three independent experiments. ${ }^{*} \mathrm{P}<0.05$ vs. CTRL; ${ }^{* *} \mathrm{P}<0.05$ vs. LPS group. CTRL, control; LPS, lipopolysaccharide; OMT, oxymatrine; FITC/FL1-H, fluorescein isothiocyanate; PI/FL2-H, propidium iodide.

A
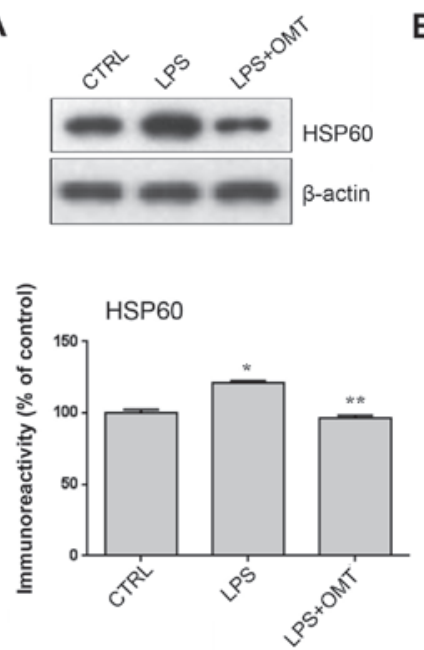

B
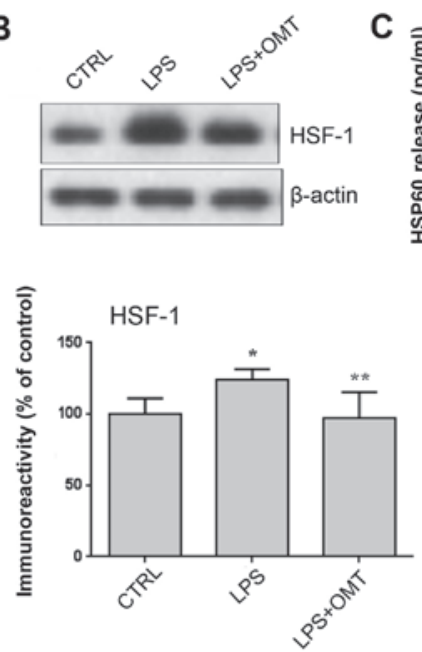

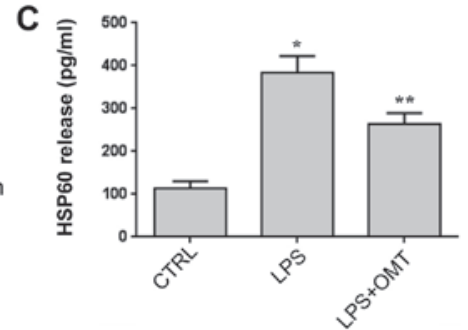

Figure 3. OMT reduces the expression and release of HSP60 and HSF-1 in LPS-stimulated BV2 microglia. BV2 cells were pre-treated with LPS for 30 min, followed by incubation with $20 \mu \mathrm{g} / \mathrm{ml}$ OMT for $24 \mathrm{~h}$. The expression levels of (A) HSP60 and (B) HSF1 were detected by western blot analysis. (C) Release of HSP60 into the supernatant was detected by ELISA. Values are expressed as the mean \pm standard error of three independent experiments. ${ }^{*} \mathrm{P}<0.05 \mathrm{vs}$. CTRL; ${ }^{* *} \mathrm{P}<0.05$ vs. LPS group. CTRL, control; LPS, lipopolysaccharide; OMT, oxymatrine; HSP, heat shock protein; HSF, heat shock factor.

by the Student-Newman-Keuls post hoc test were used for comparisons between groups. $\mathrm{P}<0.05$ was considered to indicate a statistically significant difference.

\section{Results}

OMT inhibits LPS-induced decreases of microglial cell viability. To evaluate the effects of OMT on LPS-induced toxicity on BV2 cells, the CCK-8 assay was performed (Fig. 1). After LPS stimulation, the viability of the cells was obviously decreased. Of note, at concentrations of 1-20 $\mu \mathrm{g} / \mathrm{ml}$, OMT increased the cell viability in a dose-dependent manner, indicating that it had a protective effect on BV2 cells against LPS-induced toxicity. However, compared to that in the LPS $+20 \mu \mathrm{g} / \mathrm{ml}$ OMT group, higher concentrations of OMT (50 and $100 \mu \mathrm{g} / \mathrm{ml}$ ) decreased the cell viability; therefore, the OMT concentration of $20 \mu \mathrm{g} / \mathrm{ml}$ with the maximum protective effect was used in all subsequent experiments.

OMT has anti-apoptotic effects on microglia. To further confirm the protective effect of OMT on BV2 cells, Annexin V and PI double staining followed by flow cytometric analysis 
A
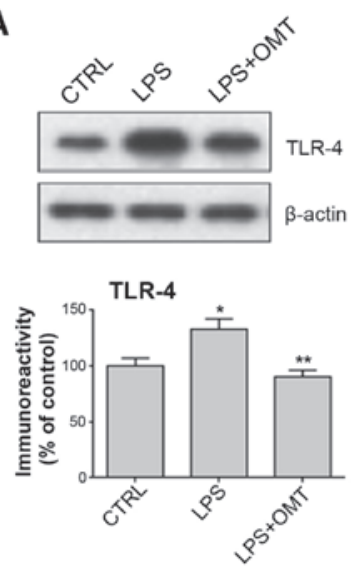

D
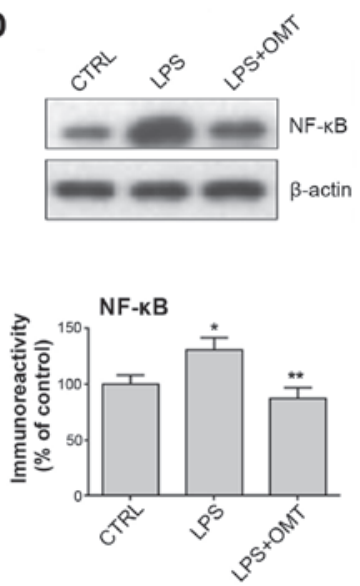

B
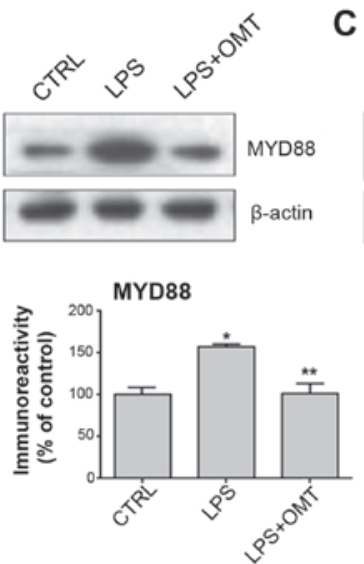

E
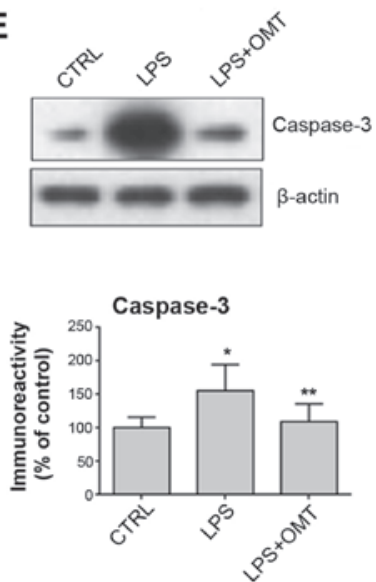

C
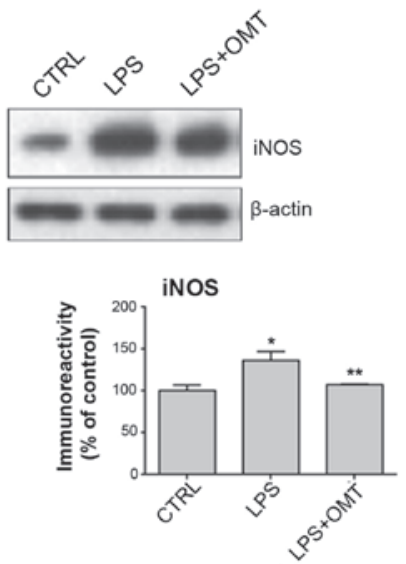

Figure 4. OMT reduces the expression of (A) TLR-4, (B) MYD88, (C) iNOS, (D) NF- $\kappa$ B and (E) caspase-3 in LPS-stimulated BV2 microglia. BV2 cells were pre-treated with LPS for $30 \mathrm{~min}$, followed by incubation with $20 \mu \mathrm{g} / \mathrm{ml}$ OMT for $24 \mathrm{~h}$. $\beta$-actin was used as a reference. Values are expressed as the mean \pm standard error of three independent experiments. ${ }^{*} \mathrm{P}<0.05$ vs. CTRL; ${ }^{* *} \mathrm{P}<0.05$ vs. LPS group. CTRL, control; LPS, lipopolysaccharide; OMT, oxymatrine; TLR, Toll-like receptor; MYD88, myeloid differentiation factor 88 ; iNOS, inducible nitric oxide synthase; NF- $\kappa \mathrm{B}$, nuclear factor- $\kappa \mathrm{B}$.

A

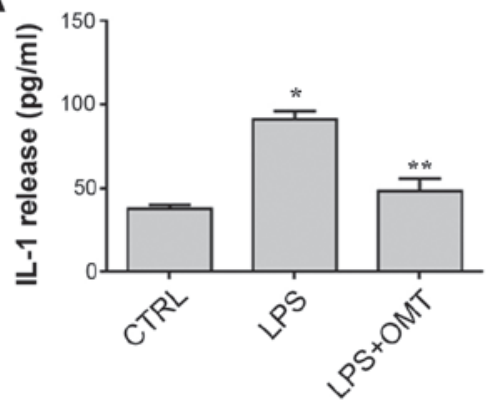

B

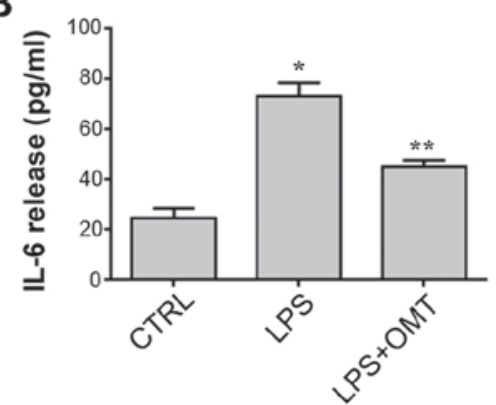

C

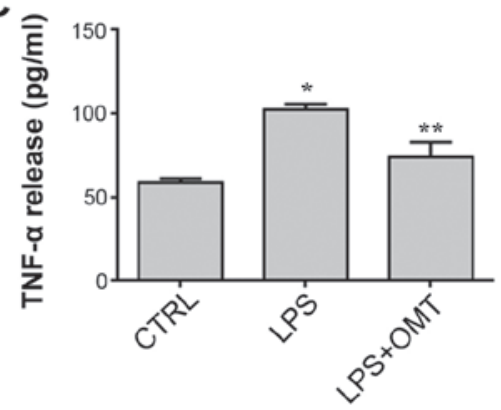

Figure 5. Effects of OMT on LPS-induced release of inflammatory cytokines in BV2 microglial cells. BV2 cells were pre-treated with LPS for 30 min, followed by incubation with $20 \mu \mathrm{g} / \mathrm{ml}$ OMT for $24 \mathrm{~h}$. The levels of (A) IL-1 $\beta$, (B) IL-6 and (C) TNF- $\alpha$ were measured by ELISA. Values are expressed as the mean \pm standard error of three independent experiments. ${ }^{*} \mathrm{P}<0.05$ vs. CTRL; ${ }^{* *} \mathrm{P}<0.05$ vs. LPS group. CTRL, control; LPS, lipopolysaccharide; OMT, oxymatrine; TNF, tumor necrosis factor; IL, interleukin.

were performed on cells pre-treated with LPS $(1 \mu \mathrm{g} / \mathrm{ml})$ for $30 \mathrm{~min}$ and incubated with $20 \mu \mathrm{g} / \mathrm{ml}$ OMT for $24 \mathrm{~h}$ (Fig. 2). The results showed that OMT significantly reduced LPS-induced apoptosis of BV2 cells. Therefore, OMT exerted a significant anti-apoptotic effect on microglia $(\mathrm{P}<0.05)$.

OMT inhibits LPS-induced expression and release of HSP60 by $B V 2$ cells. To further explore the mechanisms of the protective effects of OMT, HSP60 expression and release by BV2 cells were assessed. Numerous studies have indicated that HSP60 has a role in microglia-induced inflammation, which was confirmed by a study by our group (3). Therefore, it was hypothesized that OMT exerts its anti-inflammatory effects via HSP60. Western blot analysis showed that the expression of HSP60 was significantly increased after LPS stimulation, which was completely inhibited by OMT (Fig. 3A). HSF-1 is a transcription factor regulating HSP60 expression and was therefore examined as well. The results showed that OMT 
completely abrogated the LPS-induced increases in HSF-1 expression (Fig. 3B). Furthermore, an ELISA revealed that OMT significantly decreased the LPS-induced release of extracellular HSP60 (Fig. 3C). These results indicated that under the stress conditions, HSP60 is highly expressed and secreted into the extracellular space to induce cell apoptosis, which is consistent with the hypothesis of the present study.

OMT inhibits LPS-induced upregulation of iNOS, TLR-4, $N F-\kappa B$ p65, caspase- 3 and MYD88 expression. TLR-4 is a receptor of LPS and HSP60 on the surface of microglia, which can activate downstream signaling molecules, including NF- $\kappa$ B, caspase-3, MYD88 and iNOS. To determine whether extracellular HSP60 released by BV2 cells can bind TLR-4 and activate the TLR-4 signaling pathway and whether OMT inhibits this activation, the effects of LPS and OMT on the protein levels of TLR-4, Myd88, NF- $\kappa$ B, caspase-3 and iNOS were assessed by western blotting. The results showed that OMT abrogated the LPS-induced expression of TLR-4, NF- $\kappa$ B, caspase-3, MYD88 and iNOS in BV2 cells (Fig. 4). These experiments indicated that OMT may exert its anti-inflammatory effects via inhibiting the TLR-4 pathway.

OMT inhibits LPS-induced inflammatory cytokine release by BV2 microglial cells. Activation of the TLR-4 signaling pathway is accompanied by the production and release of inflammatory cytokines. To further investigate the effects of OMT on LPS-stimulated cytokines, the levels of TNF- $\alpha$, IL-1 $\beta$ and IL- 6 in the culture medium were measured by ELISA. As shown in Fig. 5, OMT markedly attenuated LPS-induced release of TNF- $\alpha$, IL-1 $\beta$ and IL- 6 by BV2 microglial cells. These results indicated that OMT exerts its anti-inflammatory effects by suppressing the release of inflammatory factors.

\section{Discussion}

OMT is the major alkaloid of Sophora flavescens and has anti-inflammatory, anti-allergic, anti-viral, anti-fibrotic and cardiovascular protective properties (11-13). A previous study has reported that in rats with cerebral ischemia, OMT reduced the production of $\mathrm{NF}-\kappa \mathrm{B}$ and suppressed the inflammatory reaction of the CNS to decrease the area of cerebral infarction (14). However, the neuroprotective effects of OMT and the underlying molecular mechanisms have yet to be fully elucidated. The inhibition of microglia activation has been proposed to be an effective therapeutic option for neurodegenerative diseases. Therefore, the present study investigated whether OMT treatment can inhibit microglia activation and examined the underlying mechanisms. It was revealed that OMT attenuated microglia activation by suppressing the HSP60/TLR-4/MYD88/NF-кB signaling pathway.

The mitochondrial matrix protein HSP60 can be induced by stress and forms a chaperon complex within the mitochondria, which is important for mitochondrial protein folding and function (15). Activated HSP60 mainly locates in the plasma membrane or the extracellular space and can mediate cell apoptosis. Furthermore, HSP60 has also been reported to enhance caspase activation to exert a pro-apoptotic function (16). In the present study, the effects of OMT on microglia apoptosis were assessed by Annexin V and PI double staining.
The results indicated that LPS induced apoptosis in BV2 microglial cells, which was inhibited by OMT. Further mechanistic study revealed that OMT decreased the expression and release of HSP60 triggered by LPS. It has been reported that HSP60 is a ligand of TLR-4, indicating that HSP60 evokes an immune response (17).

TLR-4 signaling may proceed via two possible pathways-the MYD88-dependent and the MYD88-independent pathway. MYD88 is the universal intracellular adaptor recruited by all known TLRs except TLR-3 (18). The results of the present study showed that the expression of MYD88 was increased in LPS-induced BV2 cells, which was abrogated by OMT, indicating that HSP60 activates microglia via the MYD88-dependent pathway. $N F-\kappa B$ is a vital factor in the TLR-4 pathway and translocates to the nucleus upon its activation, where it induces the production of several pro-inflammatory cytokines, including TNF- $\alpha$, IL- $1 \beta$ and IL-6 (19). NF- $\kappa \mathrm{B}$ is also the principal regulator of the transactivation of pro-inflammatory genes such as iNOS (20). Furthermore, $\mathrm{NF}-\kappa \mathrm{B}$ also initiates the transcription of the HSP60 gene, which leads to a potent inflammatory response in innate immune cells (21). The results of the present study showed that OMT suppressed the expression of $\mathrm{NF}-\kappa \mathrm{B}$ and iNOS in LPS-induced BV2 cells and decreased the release of inflammatory factors. The activation of NF- $\kappa \mathrm{B}$ by caspase- 3 is critical in inflammation and cell apoptosis (22). LPS-stimulated microglia have been shown not to be toxic to neighboring neurons when caspase-3 is inhibited (23). The results of the present study showed that OMT reduced the levels of cleaved caspase-3, which is consistent with the fact that OMT inhibited apoptosis, as indicated by flow cytometry.

In conclusion, the results of the present study demonstrated that OMT exerts neuroprotective and anti-inflammatory effects on LPS-induced microglial cells, which may be attributed to the inhibition of the HSP60-TLR-4-MYD88-NF- $\mathrm{B}$ pathway. Therefore, these findings provide a potential therapeutic application of OMT for neurodegenerative diseases.

\section{Acknowledgements}

The present study was supported by grants from National Natural Science Foundation of China (nos. 81460182, 31460257, 81571098, 31560273, 81260051 and 31260243) and the Ningxia Natural Science Foundation (no. NZ14057). Additional funding was provided for Dr Yin Wang by the Program for New Century Excellent Talents in University.

\section{References}

1. Kreutzberg GW: Microglia: A sensor for pathological events in the CNS. Trends Neurosci 19: 312-318, 1996.

2. Jung WK, Lee DY, Park C, Choi YH, Choi I, Park SG, Seo SK, Lee SW, Yea SS, Ahn SC, et al: Cilostazol is anti-inflammatory in BV2 microglial cells by inactivating nuclear factor-kappaB and inhibiting mitogen-activated protein kinases. $\mathrm{Br} \mathrm{J}$ Pharmacol 159: 1274-1285, 2010.

3. Li YH, Teng P, Wang Y, Zhang YM, Ma CJ and Pu J: Expression and regulation of HSP60 in activated microglia cells. J Ningxia Med Col 8: 712-714, 2011.

4. Mhatre M, Floyd RA and Hensley K: Oxidative stress and neuroinflammation in Alzheimer's disease and amyotrophic lateral sclerosis: Common links and potential therapeutic targets. J Alzheimers Dis 6: 147-157, 2004 
5. Tansey MG, McCoy MK and Frank-Cannon TC: Neuroinflammatory mechanisms in Parkinson's disease: Potential environmental triggers, pathways, and targets for early therapeutic intervention. Exp Neurol 208: 1-25, 2007.

6. McGeer PL and McGeer EG: Inflammatory processes in amyotrophic lateral sclerosis. Muscle Nerve 26: 459-470, 2002

7. Yuan X, Wang Y, Du D, Hu Z, Xu M, Xu M and Liu Z: The effects of the combination of sodium ferulate and oxymatrine on lipopolysaccharide-induced acute lung injury in mice. Inflammation 35: 1161-1168, 2012.

8. Liu Y, Xu Y, Ji W, Li X, Sun B, Gao Q and Su C: Anti-tumor activities of matrine and oxymatrine: Literature review. Tumour Biol 35: 5111-5119, 2014.

9. Cui L, Zhang X, Yang R, Wang L, Liu L, Li M and Du W: Neuroprotection and underlying mechanisms of oxymatrine in cerebral ischemia of rats. Neurol Res 33: 319-324, 2011.

10. Chandra D, Choy G and Tang DG: Cytosolic accumulation of HSP60 during apoptosis with or without apparent mitochondrial release: evidence that its pro-apoptotic or pro-survival functions involve differential interactions with caspase-3. J Biol Chem 282: 31289-31301, 2007.

11. Huang M, Hu YY, Dong XQ, Xu QP, Yu WH and Zhang ZY: The protective role of oxymatrine on neuronal cell apoptosis in the hemorrhagic rat brain. J Ethnopharmacol 143: 228 235, 2012

12. Chai NL, Fu Q, Shi H, Cai CH, Wan J, Xu SP and Wu BY: Oxymatrine liposome attenuates hepatic fibrosis via targeting hepatic stellate cells. World J Gastroenterol 18: 4199-4206, 2012.

13. Hong Li S, Lei L, Lei S, Dan Z, De Li D, Guo Fen Q, Yan L, Wen Feng $C$ and Bao Feng Y: Cardioprotective effects and underlying mechanisms of oxymatrine against ischemic myocardial injuries of rats. Phytother Res 22: 985-989, 2008.

14. Liu Y, Zhang XJ, Yang CH and Fan HG: Oxymatrine protects rat brains against permanent focal ischemia and downregulates NF-kappaB expression. Brain Res 1268: 174-180, 2009.

15. Voos W and Röttgers K: Molecular chaperones as essential mediators of mitochondrial biogenesis. Biochim Biophys Acta 1592: 51-62, 2002
16. Samali A, Cai J, Zhivotovsky B, Jones DP and Orrenius S: Presence of a pre-apoptotic complex of pro-caspase-3, Hsp60 and $\mathrm{Hsp10}$ in the mitochondrial fraction of jurkat cells. EMBO J 18: 2040-2048, 1999.

17. Lehnardt S, Schott E, Trimbuch T, Laubisch D, Krueger C, Wulczyn G, Nitsch R and Weber JR: A vicious cycle involving release of heat shock protein 60 from injured cells and activation of toll-like receptor 4 mediates neurodegeneration in the CNS. J Neurosci 28: 2320-2331, 2008.

18. Rosenberger K, Dembny P, Derkow K, Engel O, Krüger C, Wolf SA, Kettenmann H, Schott E, Meisel A and Lehnardt S: Intrathecal heat shock protein 60 mediates neurodegeneration and demyelination in the CNS through a TLR4-and MyD88-dependent pathway. Mol Neurodegener 10: 5, 2015.

19. Tsung A, McCoy SL, Klune JR, Geller DA, Billiar TR and Hefeneider SH: A novel inhibitory peptide of Toll-like receptor signaling limits lipopolysaccharide-induced production of inflammatory mediators and enhances survival in mice. Shock 27: 364-369, 2007.

20. Dasgupta S, Jana M, Zhou Y, Fung YK, Ghosh S and Pahan K: Antineuroinflammatory effect of NF-kappaB essential modifier-binding domain peptides in the adoptive transfer model of experimental allergic encephalomyelitis. J Immunol 173: 1344-1354, 2004

21. Wang Y, Chen L, Hagiwara N and Knowlton AA: Regulation of heat shock protein 60 and 72 expression in the failing heart. J Mol Cell Cardiol 48: 360-366, 2010.

22. Soria JA, Arroyo DS, Gaviglio EA, Rodriguez-Galan MC, Wang JM and Iribarren P: Interleukin 4 induces the apoptosis of mouse microglial cells by a caspase dependent mechanism. Neurobiol Dis 43: 616-624, 2011.

23. Burguillos MA, Deierborg T, Kavanagh E, Persson A, Hajji N, Garcia-Quintanilla A, Cano J, Brundin P, Englund E, Venero JL and Joseph B: Caspase signalling controls microglia activation and neurotoxicity. Nature 472: 319-324, 2011 